Pacific

Journal of

Mathematics

BOOLEAN ALGEBRAS OF PROJECTIONS \& ALGEBRAS OF SPECTRAL OPERATORS

H.R. Dowson, M.B. Ghaemi, and P.G. Spain

Volume 209 No. 1

March 2003 


\title{
BOOLEAN ALGEBRAS OF PROJECTIONS \& ALGEBRAS OF SPECTRAL OPERATORS
}

\author{
H.R. Dowson, M.B. Ghaemi, and P.G. Spain
}

\begin{abstract}
We show that, given a weak compactness condition which is always satisfied when the underlying space does not contain an isomorphic copy of $c_{0}$, all the operators in the weakly closed algebra generated by the real and imaginary parts of a family of commuting scalar-type spectral operators on a Banach space will again be scalar-type spectral operators, provided that (and this is a necessary condition with even only two operators) the Boolean algebra of projections generated by their resolutions of the identity is uniformly bounded.
\end{abstract}

\section{Introduction.}

The problem we address, raised by Dunford [8] in 1954, is to find conditions under which the sum and product of a pair of commuting scalar-type spectral operators on a Banach space is also a scalar-type spectral operator.

Two difficulties arise when working on an arbitrary Banach space, as opposed to a Hilbert space: the unit ball of the algebra of bounded linear operators need not be weakly compact; and the Boolean algebra generated by two uniformly bounded Boolean algebras of projections need not be bounded [15].

In view of this we must restrict ourselves to the case where the Boolean algebra generated by the resolutions of the identities is uniformly bounded.

Previous treatments of this problem [to show that the sum of two commuting scalar-type spectral operators is a scalar-type spectral operator] have focussed on identifying the resolution of the identity of the sum $[\mathbf{1 1}, \mathbf{1 6}, \mathbf{2 0}]$. These methods have worked essentially only when $X$ contains no copy of $c_{0}$. However, this is precisely the case when one can exploit Grothendieck's theorem on the automatic weak compactness of linear mappings from a $\mathrm{C}^{*}$ algebra into $X$, and prove somewhat more: that all operators in the weakly closed involutory algebra generated by them are scalar-type spectral operators. An advantage of this approach is that one does not have to identify the resolutions of the identity of the sums, or products, or limits, directly. 


\section{2. $\mathrm{C}^{*}$-algebras on Banach spaces.}

The properties of scalar-type spectral operators and the involutory algebras they generate seem best explained in the context of numerical range, of hermitian operators, and of $\mathrm{C}^{*}$-algebras. For the sake of completeness, and the convenience of the reader, we present a résumé of the key results.

Consider a complex Banach space $X$; write $L(X)$ for the Banach algebra of bounded linear operators on $X$, endowed with the operator norm.

We write $A_{1}$ for the unit ball of a subset $A$ of a normed space.

We write $\left\langle x, x^{\prime}\right\rangle$ for the value of the functional $x^{\prime}$ in $X^{\prime}$ at $x$ in $X$. Let $\boldsymbol{\omega}$ be the linear span of the functionals $\omega_{x, x^{\prime}}: L(X) \rightarrow \mathbb{C}: T \mapsto\left\langle T x, x^{\prime}\right\rangle$. Let $\Pi$ be the set

$$
\left\{\left(x, x^{\prime}\right) \in X \times X^{\prime}:\left\langle x, x^{\prime}\right\rangle=\|x\|=\left\|x^{\prime}\right\|=1\right\}
$$

and let $\boldsymbol{\omega}_{\Pi}$ be the set of functionals

$$
\left\{\omega_{x, x^{\prime}}:\left(x, x^{\prime}\right) \in \Pi\right\} .
$$

The strong operator topology and weak operator topology on $L(X)$ are of paramount importance: important here too are the BWO topology and BSO topology, the strongest topologies coinciding with the weak and strong topologies on bounded subsets of $L(X)$ - see [9, VI, 9].

The ultraweak operator topology on $L(X)$ is the topology generated by the seminorms $T \mapsto\left|\sum_{n}\left\langle T x_{n}, x_{n}^{\prime}\right\rangle\right|$ where $\left\{x_{n}\right\}$ and $\left\{x_{n}^{\prime}\right\}$ range over pairs of sequences in $X$ and $X^{\prime}$ subject to $\sum_{n}\left\|x_{n}\right\|\left\|x_{n}^{\prime}\right\|<\infty$. The ultrastrong operator topology on $L(H)$ is the topology generated by the seminorms $T \mapsto$ $\left\{\sum_{n}\left\|T x_{n}\right\|^{2}\right\}^{\frac{1}{2}}$ where $\left\{x_{n}\right\}$ ranges over sequences for which $\sum_{n}\left\|x_{n}\right\|^{2}<\infty$.

The BWO topology coincides with the ultraweak topology, the BSO topology with the ultrastrong topology, on $L(H)$, when $H$ is a Hilbert space.

The (spatial) numerical range $V(T)$ of an operator $T$ is defined to be

$$
V(T) \triangleq\left\{\left\langle T x, x^{\prime}\right\rangle:\left(x, x^{\prime}\right) \in \Pi\right\} .
$$

An operator $R$ on $X$ is hermitian if its numerical range is real i.e., if $V(R) \subset \mathbb{R}$; equivalently, if

$$
\{\|\exp (i r R)\|: r \in \mathbb{R}\}
$$

is bounded. The set of hermitian operators is closed in the norm, strong and weak operator topologies.

The following result is crucial:

Theorem 2.1 (Vidav-Palmer Theorem). Suppose that $\mathcal{A}$ is a unital subalgebra of $L(X)$ [the unit being the identity operator on $X]$. Let $\mathcal{H}$ be the set of hermitian elements of $\mathcal{A}$. Then $\mathcal{A}=\mathcal{H}+i \mathcal{H}$ if and only if $\mathcal{A}$ is a pre- $\mathrm{C}^{*}$-algebra under the operator norm and the natural involution

$$
*: \mathcal{A} \rightarrow \mathcal{A}: R+i J \mapsto R-i J \quad(R, J \in \mathcal{H}) .
$$


It then follows that $\mathcal{B} \triangleq \overline{\mathcal{A}}$ is a $\mathrm{C}^{*}$-algebra on $X$, containing the identity $I_{X}$ on $X$. (See $[\mathbf{3}, \S 38]$ for a discussion of these topics.)

When $\mathcal{B}$ is a $\mathrm{C}^{*}$-algebra on $X$ the family $\boldsymbol{\omega}_{\Pi}$ is a separating family of states on $\mathcal{B}$.

We shall use the following terminology: a von Neumann algebra is a weakly closed $\mathrm{C}^{*}$-algebra of operators on a Hilbert space, while a $\mathrm{W}^{*}$-algebra is a $\mathrm{C}^{*}$-algebra which has a realisation as a von Neumann algebra [equivalently, is a dual space of a Banach space].

Unital *-isomorphisms of $\mathrm{C}^{*}$-algebras are isometric.

Theorem 2.2 (BWO Closure Theorem). Suppose that $\mathcal{B}$ is a $\mathrm{C}^{*}$-algebra on $X$ and that its unit ball $\mathcal{B}_{1}$ is relatively weakly compact. Then the $\mathrm{BWO}$ closure of $\mathcal{B}$,

$$
\mathcal{B}^{\sim} \triangleq \bigcup_{n=1}^{\infty} n \overline{\mathcal{B}}^{w}
$$

is a $\mathrm{W}^{*}$-algebra; and $\left(\mathcal{B}^{\Upsilon}\right)_{1}=\overline{\mathcal{B}}_{1}{ }^{w}$. Moreover, any faithful representation of $\mathcal{B}^{\sim}$ as a concrete von Neumann algebra is BWO bicontinuous.

The proof $[\mathbf{2 4}]$ rests on the fact that, by the identity of comparable compact Hausdorff topologies, the weak topology on ${\overline{\mathcal{B}_{1}}}^{w}$ is the weak topology induced by the states $\boldsymbol{\omega}_{\Pi}$.

It remains open, in general, to decide whether $\mathcal{B}^{\sim}=\overline{\mathcal{B}}^{w}$.

2.1. Commutative $\mathbf{C}^{*}$-algebras on $X$. The remaining results in this section apply to any commutative unital $\mathrm{C}^{*}$-subalgebra $\mathcal{B}$ of $L(X)$, and in particular to any algebra generated by a Boolean algebra of (hermitian) projections: see $\S 3$.

The operators in a commutative $\mathrm{C}^{*}$-subalgebra of $L(X)$ are called normal (sometimes strongly normal). Abstractly, they enjoy all the properties of normal operators on Hilbert spaces.

Let $\Lambda$ be the maximal ideal space of $\mathcal{B}$ and $\Theta$ the inverse Gelfand map

$$
\Theta: C(\Lambda) \rightarrow \mathcal{B}
$$

which is a unital isometric *-isomorphism: $\Theta$ is also called the functional calculus for $\mathcal{B}$.

On restricting $\Theta$ to the $\mathrm{C}^{*}$-subalgebra generated by $I, T$ (for any $T \in \mathcal{B}$ ) we obtain a functional calculus for a (strongly) normal $T$ : a unital isometric *-isomorphism

$$
\Theta_{T}: C(\operatorname{sp}(T)) \rightarrow \mathcal{B}
$$


such that

$$
\begin{aligned}
\Theta_{T}(z \mapsto 1) & =I \\
\Theta_{T}(z \mapsto z) & =T \\
\Theta_{T}(z \mapsto \bar{z}) & =T^{*} \\
\left\|\Theta_{T}(f)\right\| & =\|f\|_{\mathrm{sp}(T)} \quad(f \in C(\operatorname{sp}(T))) .
\end{aligned}
$$

The following two lemmas demonstrate how to some extent normal operators on a Banach space mimic normal operators on a Hilbert space:

Lemma 2.3. Let $\mathcal{B}$ be a commutative $\mathrm{C}^{*}$-algebra on $X$ and let $\mathcal{H}$ be the set of hermitian elements of $\mathcal{B}$. Suppose that ${ }_{K}^{H} \in \mathcal{H}$ and $0 \leq H \leq K$. Then

$$
\|H x\| \leq\|K x\| \quad(x \in X) .
$$

For any $\varepsilon>0$ the operator $L=H /(K+\varepsilon I)$ is defined in $\mathcal{H}$, and, by the functional calculus, $0 \leq L \leq 1$; so $\|L\| \leq 1$. It follows that $\|H x\|=$ $\|L(K+\varepsilon I) x\| \leq\|(K+\varepsilon I) x\|$. Now let $\varepsilon \rightarrow 0$.

The next result, originally due to Palmer [18, Lemma 2.7], helps us extend the $\mathrm{C}^{*}$ structure from $\mathcal{B}$ to $\mathcal{C} \triangleq \overline{\mathcal{B}}^{w}$. The following short proof is taken from [4]:

Lemma 2.4. For all $B \in \mathcal{B}$ and $x \in X$ we have

$$
\|B x\|=\left\|B^{*} x\right\| \text {. }
$$

Proof. For $\varepsilon>0$ the functional calculus gives

$$
\left\|B-B^{2}\left(B^{*} B+\varepsilon I\right)^{-1} B^{*}\right\|=\left\|\varepsilon B\left(B^{*} B+\varepsilon I\right)^{-1}\right\| \leq \sqrt{\varepsilon} / 2,
$$

and

$$
\left\|B^{2}\left(B^{*} B+\varepsilon I\right)^{-1}\right\| \leq 1
$$

Thus, for any $x \in X$,

$$
\|B x\|=\lim _{\varepsilon \rightarrow 0}\left\|B^{2}\left(B^{*} B+\varepsilon I\right)^{-1} B^{*} x\right\| \leq\left\|B^{*} x\right\|,
$$

and then $\left\|B^{*} x\right\| \leq\left\|B^{* *} x\right\|=\|B x\|$.

The weak closure of a commutative $\mathrm{C}^{*}$-algebra on $X$ is also a $\mathrm{C}^{*}$-algebra on $X$.

Theorem 2.5. Let $\mathcal{B}$ be a commutative $\mathrm{C}^{*}$-algebra on $X$ and let $\mathcal{H}$ be the set of hermitian elements of $\mathcal{B}$. Let $\overline{\mathcal{H}}^{w}$ be the weak operator topology closure of $\mathcal{H}$, and $\overline{\mathcal{B}}^{w}$ the weak operator topology closure of $\mathcal{B}$. Then

$$
\overline{\mathcal{B}}^{w}=\overline{\mathcal{H}}^{w}+i \overline{\mathcal{H}}^{w}
$$

and so $\overline{\mathcal{B}}^{w}$ is a $\mathrm{C}^{*}$-algebra. Moreover, $\left(\overline{\mathcal{B}}^{w}\right)_{1}=\overline{\mathcal{B}}_{1}{ }^{w}$. Hence $\mathcal{B}^{\sim}=\overline{\mathcal{B}}^{w}$. 
Proof. First note that the weak and strong closures coincide for $\mathcal{H}$ and $\mathcal{B}$ (they are both convex sets). Now Lemma 2.4 shows that $\overline{\mathcal{B}}^{s}=\overline{\mathcal{H}}^{s}+i \overline{\mathcal{H}}^{s}$, so $\overline{\mathcal{B}}^{w}$ is a $\mathrm{C}^{*}$-algebra.

Consider $H \in\left(\overline{\mathcal{H}}^{w}\right)_{1}$. Then $K=\left(I-\left[I-H^{2}\right]^{\frac{1}{2}}\right) / H \in \overline{\mathcal{H}}^{w}$, and $H=$ $2 K /\left(I+K^{2}\right)$. Take a net $K_{\alpha}$ in $\mathcal{H}$ converging strongly to $K$ : put $H_{\alpha}=$ $2 K_{\alpha} /\left(I+K_{\alpha}^{2}\right)$. Then

$$
H_{\alpha}-H=2\left(I+K_{\alpha}^{2}\right)^{-1}\left(K_{\alpha}-K\right)\left(I+K^{2}\right)^{-1}+\frac{1}{2} H_{\alpha}\left(K-K_{\alpha}\right) H
$$

so $H \in{\overline{\mathcal{H}_{1}}}^{w}$. By the Russo-Dye Theorem $[3, \S 38]$ we have $\left(\overline{\mathcal{B}}^{w}\right)_{1} \subseteq \overline{\mathcal{B}}_{1}$.

Corollary 2.6. If, further, the unit ball of $\mathcal{B}$ is relatively weakly compact, then $\overline{\mathcal{B}}^{w}$ is a $\mathrm{W}^{*}$-algebra and any faithful representation of $\overline{\mathcal{B}}^{w}$ as a concrete von Neumann algebra on a Hilbert space is BWO bicontinuous (that is, weakly bicontinuous on bounded sets).

Proof. Use Theorem 2.2.

Remark 2.7. We show later $(\S 4)$ that any such faithful representation is also BSO bicontinuous (that is, strongly bicontinuous on bounded sets). The proof (maybe the result) depends on being able to represent $\overline{\mathcal{B}}^{w}$ by a spectral measure: and the presence of $c_{0}$ as a subspace of $X$ seems to be the natural obstruction to this: see $\S 6$ below.

\section{Boolean algebras of projections \& the algebras they generate.}

Let $X$ be a complex Banach space, and $\mathcal{E}$ a bounded Boolean algebra of projections on $X$ :

$$
\begin{aligned}
& I \in \mathcal{E} \quad \subseteq \quad L(X) \\
& E \in \mathcal{E} \quad \Longrightarrow \quad E^{2}=E \\
& E \in \mathcal{E} \quad \Longrightarrow \quad I-E \in \mathcal{E} \\
& E, F \in \mathcal{E} \quad \Longrightarrow \quad E F=F E \in \mathcal{E} \\
& \|E\| \leq K_{\mathcal{E}} \quad(E \in \mathcal{E})
\end{aligned}
$$

for some constant $K_{\mathcal{E}}$. Write aco $\mathcal{E}$ for the absolutely convex hull of $\mathcal{E}$ in $L(X)$.

It can be shown (see $[6,5.4])$ that then

$$
\mathcal{S}=\left\{\sum_{\text {finite }} \lambda_{j} E_{j}:\left|\lambda_{j}\right| \leq 1, E_{j} \in \mathcal{E}, E_{j} E_{k}=0(j \neq k)\right\}
$$

is a bounded multiplicative semigroup of operators on $X$. If we define

$$
\|x\|_{\mathcal{E}}=\sup \{\|S x\|: S \in \mathcal{S}\} \quad(x \in X)
$$


we obtain a norm $\|\cdot\|_{\mathcal{E}}$ on $X$, equivalent to the original norm on $X$, with respect to which each element of $\mathcal{E}$ is hermitian. Thus, without loss of generality,

we shall assume that all elements of $\mathcal{E}$ are hermitian.

Remark 3.1. By Sinclair's Theorem $\|E\|=1$ for any nonzero hermitian projection.

Theorem 3.2. Let $\mathcal{E}$ be a Boolean algebra of hermitian projections on a complex Banach space $X$. Then $\mathcal{A}$, the linear span of $\mathcal{E}$, is the *algebra generated by $\mathcal{E}: \mathcal{A}$ is a commutative unital algebra, and $\mathcal{A}=\mathcal{H}+i \mathcal{H}$, where $\mathcal{H}$ is the set of hermitian elements of $\mathcal{A}$. So $\mathcal{B}, \triangleq \overline{\mathcal{A}}$, is a commutative $\mathrm{C}^{*}$-algebra on $X$.

Proof. Immediate from the Vidav-Palmer Theorem (Theorem 2.1).

Lemma 3.3. Let $S \in \mathcal{A}$ and suppose that $-I \leq S \leq I$. Then

$$
S \in 2 \operatorname{aco} \mathcal{E} \text {. }
$$

Proof. Suppose first that $0 \leq S \leq I$. Write $S$ in $\mathcal{E}$-step-form as $S=$ $\sum_{j=1}^{M} \lambda_{j} E_{j}$, where the $E_{j}$ are pairwise disjoint. Then $0 \leq \lambda_{j} \leq 1$. Arrange the $\lambda_{j}$ in descending order: then $\|S\|=\lambda_{1}$. Define $\lambda_{M+1}=0$ and use Abel summation -

$$
S=\sum_{j=1}^{M} \lambda_{j} E_{j}=\sum_{j=1}^{M}\left(\lambda_{j}-\lambda_{j+1}\right)\left(\sum_{h=1}^{j} E_{h}\right) \in \operatorname{aco} \mathcal{E} .
$$

If $-I \leq S \leq I$, split $S$ into its positive and negative parts.

Theorem 3.4. Let $\mathcal{E}$ be a Boolean algebra of hermitian projections on a complex Banach space $X$, and let $\mathcal{B}$ be the $\mathrm{C}^{*}$-algebra it generates. Let $\mathcal{B}_{1}$ be the closed unit ball of $\mathcal{B}$. Then

$$
\mathcal{B}_{1} \subseteq 4 \overline{\operatorname{aco}} \mathcal{E} .
$$

Proof. Consider an element $B \in \mathcal{B}$ such that $\|B\|<1$. Given $\varepsilon>0$ we can find $S=R+i J$ in $\mathcal{A}$ such that $\|B-R-i J\| \leq \min \{\varepsilon, 1-\|B\|\}$. Now $\|R\| \leq 1$, so that, by Lemma $3.3,{ }_{J}^{R} \in 2$ aco $\mathcal{E}$.

Corollary 3.5. The following are equivalent:

1) $\mathcal{B}_{1}$ is relatively weakly compact.

2) aco $\mathcal{E}$ is relatively weakly compact.

3) $\mathcal{E}$ is relatively weakly compact.

Proof. Use the Krein-Šmulian Theorem. 
We can now state the main theorem of this section.

Theorem 3.6. Let $\mathcal{E}$ be a relatively weakly compact Boolean algebra of hermitian projections on a complex Banach space $X$, and let $\mathcal{B}$ be the $\mathrm{C}^{*}$-algebra generated by $\mathcal{E}$. Then $\overline{\mathcal{B}}^{w}$ is a $\mathrm{W}^{*}$-algebra and any faithful representation of $\overline{\mathcal{B}}^{w}$ as a concrete von Neumann algebra on a Hilbert space is BWO bicontinuous (that is, weakly bicontinuous on bounded sets).

Proof. This follows from Corollary 3.5 and Theorem 2.2.

\section{4. $\sigma$-complete Boolean algebras of projections \& spectral measures.}

The fundamental results on Boolean algebras of projections on a Banach space were developed by Bade and are to be found in [10, XVII]. Much interesting material on this topic is also to be found in [21].

Following [10] we say that an abstract Boolean algebra $\mathcal{E}$ is $(\sigma-)$ complete if each (countable) subset of $\mathcal{E}$ has a supremum and infimum in $\mathcal{E}$.

$\mathcal{E}$, a Boolean algebra of projections on $X$, is $(\sigma-)$ complete on $X$ if each (countable) subset $\mathcal{F}$ of $\mathcal{E}$ has a supremum and infimum in $\mathcal{E}$ such that

$$
(\bigvee \mathcal{F}) X=\varlimsup \operatorname{lin}\{F X: F \in \mathcal{F}\}, \quad(\bigwedge \mathcal{F}) X=\bigcap_{F \in \mathcal{F}} F X .
$$

It has been shown that $\mathcal{E}$ is $(\sigma$-)complete on $X$ if and only if every bounded monotone (sequence) net in $\mathcal{E}$ converges strongly to a limit [10, XVII.3.4]. In this case $\mathcal{E}$ must be bounded [10, XVII.3.3].

On Hilbert space. On a Hilbert space $\mathcal{H}$ the following two facts are classical. We sketch their (elementary) proofs for the convenience of the reader.

Fact 4.1. Any monotone net of hermitian projections on $\mathcal{H}$ has a supremum, to which it converges strongly.

Proof. Let $\left(E_{\alpha}\right)_{\alpha \in A}$ be such a net. The generalized Cauchy-Schwarz inequality $\left\langle P^{2} \xi, \xi\right\rangle \leq\langle P \xi, \xi\rangle\left\langle P^{3} \xi, \xi\right\rangle$, which holds for any positive operator $P$ on $\mathcal{H}$ and any element $\xi \in \mathcal{H}$, shows that the net $\left(E_{\alpha}\right)_{\alpha \in A}$ is strongly Cauchy. Also, its limit must be the supremum.

Fact 4.2. Suppose that $\left(E_{\alpha}\right)_{\alpha \in A}$ is a net of hermitian projections that converges weakly to a projection $E$. Then it converges strongly.

Proof. This is immediate from the calculation

$$
\begin{aligned}
\left\|\left(E-E_{\alpha}\right) \xi\right\|^{2} & =\left\langle\left(E-E_{\alpha}\right)^{2} \xi, \xi\right\rangle \\
& =\left\langle E^{2} \xi, \xi\right\rangle-\left\langle E E_{\alpha} \xi, \xi\right\rangle-\left\langle E_{\alpha} E \xi, \xi\right\rangle+\left\langle E_{\alpha}^{2} \xi, \xi\right\rangle \\
& \rightarrow\left\langle\left(E-E^{2}\right) \xi, \xi\right\rangle=0 .
\end{aligned}
$$


It follows that on a Hilbert space every Boolean algebra $\mathcal{E}$ of hermitian projections can be extended to a complete one; that $\overline{\mathcal{E}}^{s}$ is the smallest such complete extension; and that $\overline{\mathcal{E}}^{s}=\overline{\mathcal{E}}^{w} \bigcap\{$ projections on $\mathcal{H}\}$.

On a Banach space the situation is more delicate. It has been shown that if $\mathcal{E}$ is $\sigma$-complete on $X$ then $\overline{\mathcal{E}}^{s}$ is complete on $X$ [10, XVII.3.23], and that the family of projections in $\overline{\mathcal{E}}^{w}$ coincides with $\overline{\mathcal{E}}^{s}$. See Corollary 4.10 below for a proof [independent of Bade's original methods].

We shall require the following result, proposed as an exercise in [9]:

Lemma 4.3. If $\mathcal{S} \subset L(X)$ then $\mathcal{S}$ is relatively compact in the weak operator topology if and only if the sets $\mathcal{S} x$ are relatively weakly compact for all $x \in X$.

Proof. See [9, VI.9.2].

4.1. Spectral measures. Let $\Sigma$ be a $\sigma$-algebra of subsets of a set $\Omega$ and $\Gamma$ a total subset of $X^{\prime}$. A spectral measure of class $(\Sigma, \Gamma)$ is a Boolean algebra homomorphism $\sigma \mapsto E(\sigma)$ from $\Sigma$ into $L(X)$ such that $\left\langle E(\sigma) x, x^{\prime}\right\rangle$ is countably additive for each $x \in X$ and $x^{\prime} \in \Gamma$ : by the Banach-Orlicz-Pettis theorem any spectral measure of class $X^{\prime}$ is strongly countably additive.

A $\sigma$-complete Boolean algebra of projections $\mathcal{E}$ on $X$ can be identified with the range of a spectral measure of class $X^{\prime}$ on the Borel sets of the Stone space of $\mathcal{E}([\mathbf{5}$, Chapter $\mathrm{I}])$ : then each vector measure $\mathcal{E} x$ is strongly countably additive.

Lemma 4.4. If $\mu$ is a strongly countably additive vector measure with values in $X$ then $\operatorname{aco}\{\mu(\sigma): \sigma \in \Sigma\}$ is relatively weakly compact.

Proof. Essentially this is a result of Bartle, Dunford and Schwartz [1, 2.3]: see also [5, I.2.7 \& I.5.3].

Corollary 4.5. If $\mathcal{E}$ is $\sigma$-complete then the set $\overline{\mathrm{aco}}^{w}(\mathcal{E} x)$ is weakly compact for each $x \in X$.

Theorem 4.6. Let $\mathcal{E}$ be a $\sigma$-complete Boolean algebra of hermitian projections. Then $\mathcal{C}, \triangleq \overline{\mathcal{B}}^{w}$, the commutative $\mathrm{C}^{*}$-algebra generated by $\mathcal{E}$ in the weak operator topology, is a $\mathrm{W}^{*}$-algebra, and $\mathcal{C}_{1}=\overline{\mathcal{B}}_{1}{ }^{w} \subseteq 4 \overline{\mathrm{aco}}^{w} \mathcal{E}$. Furthermore, any faithful representation of $\mathcal{C}$ as a von Neumann algebra on a Hilbert space is weakly bicontinuous on bounded sets.

Proof. $\overline{\operatorname{aco}}^{w}(\mathcal{E} x)$ is weakly compact for each $x \in X$ (Corollary 4.5) so aco $(\mathcal{E})$ is relatively weakly compact, by Lemma 4.3. Apply Theorem 3.6.

Theorem 4.7. Let $\mathcal{B}$ be a commutative $\mathrm{C}^{*}$-algebra on $X$ such that $\mathcal{B}_{1}$ is relatively weakly compact. Let $\mathcal{C}=\overline{\mathcal{B}}^{w}$. Then there is a representing spectral measure $E(\cdot)$ defined on the Borel sets of the Gelfand space $\Lambda$ of $\mathcal{C}$ such that

$$
\Theta(f)=\int_{\Lambda} f(\lambda) E(d \lambda) \quad(f \in C(\Lambda)) .
$$


Proof. Let $\pi: \mathcal{C} \rightarrow L(H)$ be a BWO continuous representation of $\mathcal{C}$ as a concrete $\mathrm{W}^{*}$-algebra. Let $\widetilde{E}(\cdot)$ be a representing spectral measure for $\pi(\mathcal{C})$ :

$$
\pi \circ \Theta(f)=\int_{\Lambda} f(\lambda) \widetilde{E}(d \lambda) \quad(f \in C(\Lambda)) .
$$

Now define $E(\cdot)=\pi^{-1} \widetilde{E}(\cdot)$ : this yields a spectral measure on $X[E(\cdot)$ is weakly countably additive, and so, by the Banach-Orlicz-Pettis theorem, strongly countably additive]: and then

$$
\Theta(f)=\int_{\Lambda} f(\lambda) E(d \lambda) \quad(f \in C(\Lambda))
$$

It is immediate that for a bounded net $\left(T_{\alpha}\right)_{\alpha \in A}$ of operators on a Hilbert space we have

$$
\left(T_{\alpha}\right)_{\alpha \in A} \rightarrow_{\text {strongly }} 0 \Longleftrightarrow\left(T_{\alpha}^{*} T_{\alpha}\right)_{\alpha \in A} \rightarrow_{\text {weakly }} 0 .
$$

A similar result holds for normal operators on a Banach space provided that they belong to a common $\mathrm{W}^{*}$-algebra.

Theorem 4.8. Let $\mathcal{C}$ be a commutative $\mathrm{W}^{*}$-algebra on $X$. Suppose that $\left(S_{\alpha}\right)_{\alpha \in A}$ is a bounded net in $\mathcal{C}$. Then

$$
\left(S_{\alpha}\right)_{\alpha \in A} \rightarrow_{\text {strongly }} 0 \Longleftrightarrow\left(S_{\alpha}^{*} S_{\alpha}\right)_{\alpha \in A} \rightarrow_{\text {weakly }} 0 .
$$

Proof. Clearly $S_{\alpha} \rightarrow_{\text {strongly }} 0$ implies that $S_{\alpha}^{*} S_{\alpha} \rightarrow_{\text {strongly }} 0$, whence $S_{\alpha}^{*} S_{\alpha}$ $\rightarrow$ weakly 0 .

Let $E(\cdot)$ be the representing spectral measure for $\mathcal{C}$ guaranteed by Theorem 4.7 .

Suppose that $S_{\alpha}^{*} S_{\alpha} \rightarrow_{\text {weakly }} 0$. Let $f_{\alpha}=\Theta^{-1} S_{\alpha}$. Then

$$
\lim _{\alpha}\left\langle S_{\alpha}^{*} S_{\alpha} x, x^{\prime}\right\rangle=\lim _{\alpha} \int_{\Lambda}\left|f_{\alpha}\right|^{2}\left\langle E(d \lambda) x, x^{\prime}\right\rangle \quad\left(x \in X, x^{\prime} \in X^{\prime}\right) .
$$

Therefore $\lim _{\alpha} f_{\alpha}=0$ in $\operatorname{var}\left\langle E(\cdot) x, x^{\prime}\right\rangle$ measure and $\lim _{\alpha} \int_{\Lambda} f_{\alpha}\left\langle E(d \lambda) x, x^{\prime}\right\rangle=$ 0 . For fixed $x \in X$ the set $\left\{\left\langle E(\cdot) x, x^{\prime}\right\rangle:\left\|x^{\prime}\right\| \leq 1\right\}$ is a relatively weakly compact set of measures [9, IV.10.2]: hence $\lim _{\alpha} \int_{\Lambda} f_{\alpha}\left\langle E(d \lambda) x, x^{\prime}\right\rangle=0$ uniformly for $\left\|x^{\prime}\right\| \leq 1\left[\mathbf{1 4}\right.$, Théorème 2]. Therefore $\lim _{\alpha} \int_{\Lambda} f_{\alpha} E(d \lambda) x=0$; that is, $S_{\alpha} \rightarrow$ strongly 0 .

Corollary 4.9. Let $\mathcal{C}$ be a commutative $\mathrm{W}^{*}$-algebra on $X$. Then any faithful concrete representation of $\mathcal{C}$ as a von Neumann algebra is weakly and strongly bicontinuous on bounded sets.

Corollary 4.10. Let $\mathcal{E}$ be a $\sigma$-complete Boolean algebra of hermitian projections, and let $\left(E_{\alpha}\right)_{\alpha \in A}$ be a monotone net of hermitian projections in 
the commutative $\mathrm{W}^{*}$-algebra $\mathcal{C}$ generated on $X$ by $\mathcal{E}$. Then $\left(E_{\alpha}\right)_{\alpha \in A}$ converges strongly to a projection in $\mathcal{C}$. So $\overline{\mathcal{E}}^{s}$ is complete on $X$. What is more, $\overline{\mathcal{E}}^{s}=\overline{\mathcal{E}}^{w} \bigcap\{$ projections in $\mathcal{C}\}$.

Proof. This follows immediately from the known results on Hilbert spaces and from the strong bicontinuity of faithful representations guaranteed by the theorem.

The next corollary complements $[\mathbf{2 3}$, Theorem 5] and [12, Theorems 1 , 2].

Corollary 4.11. Let $\mathcal{E}$ be a bounded Boolean algebra of projections on a Banach space $X$ and suppose that $\mathcal{E}$ is relatively weakly compact. Then $\mathcal{E}$ has a $(\sigma-)$ complete extension contained in $\overline{\mathcal{E}}^{s}$.

Remark 4.12. This happens automatically when $X \not \supset c_{0}$ (see $\S 6$ ).

Corollary 4.13 ([10, XVII.3.7]). Let $\mathcal{E}$ be a complete bounded Boolean algebra of projections on a Banach space $X$. Then $\mathcal{E}$ is strongly closed.

Remark 4.14. The results of [7] overlap with ours.

\section{Spectral operators.}

An operator $T \in L(X)$ is prespectral of class $\Gamma$ if there is a spectral measure $E(\cdot)$ of class $\left(\Sigma_{p}, \Gamma\right)$ (here $\Sigma_{p}$ is the family of Borel subsets of the complex plane) such that for all $\sigma \in \Sigma_{p}$ :

$$
\begin{aligned}
& T E(\sigma)=E(\sigma) T, \\
& \operatorname{sp}(T \mid E(\sigma) X) \subseteq \bar{\sigma} .
\end{aligned}
$$

The spectral measure $E(\cdot)$ is called a resolution of the identity of class $\Gamma$ for $T$. If, further, $T=\int_{\operatorname{sp}(T)} \lambda E(d \lambda)$, then $T$ is a scalar-type operator of class $\Gamma$.

Remark 5.1. Given a scalar-type spectral operator $T=\int_{\mathrm{sp}(T)} \lambda E(d \lambda)$ we can define its real part $\Re T=\int_{\mathrm{sp}(T)} \Re \lambda E(d \lambda)$, and its imaginary part $\Im T=\int_{\operatorname{sp}(T)} \Im \lambda E(d \lambda)$. By the (closed) *-algebra generated by $T$ we mean the (closed) algebra generated by $\Re T$ and $\Im T$.

An operator $T \in L(X)$ is a spectral operator if it is prespectral of class $X^{\prime}$ : that is, if there is a spectral measure $E(\cdot)$ of class $X^{\prime}$ satisfying Conditions (1) and (2) above, and if also

$E(\cdot)$ is strongly countably additive on $\Sigma_{p}$.

An important property of spectral operators is that if $T$ is spectral and $S$ commutes with $T$, then $S$ commutes with the resolution of the identity of $T$ [6, Theorem 6.6].

Scalar-type spectral operators have been characterised as follows: 
Theorem $5.2([\mathbf{1 7}] \&[\mathbf{2 2}$, Theorem $])$. The operator $T \in L(X)$ is a scalartype spectral operator if and only if it satisfies the following two conditions:

(1) $T$ has a functional calculus, and

(2) for every $x \in X$ the map $\Theta_{x}: C(\operatorname{sp}(T)) \rightarrow X: f \mapsto \Theta(f) x$ is weakly compact.

Note that by Lemma 4.3 Property (2) is equivalent to:

$\left(2^{\prime}\right)$ The functional calculus $\Theta: C(\operatorname{sp}(T)) \rightarrow L(X)$ is weakly compact in the sense that $\Theta\left(\left\{f \in C(\operatorname{sp}(T)):\|f\|_{\mathrm{sp}(T)} \leq 1\right\}\right)$ is relatively compact in the weak operator topology of $L(X)$.

\section{In the absence of $c_{0}$.}

The following theorem goes back to Grothendieck, Bartle-Dunford-Schwartz, and others. See [5, VI, Notes] for an interesting discussion of its genesis and development.

Theorem 6.1. If $\mathcal{B}$ is a $\mathrm{C}^{*}$-algebra, if $\Theta: \mathcal{B} \rightarrow X$ is a bounded operator, and $X$ does not contain a subspace isomorphic to $c_{0}$, then $\Theta$ is a weakly compact mapping.

Remarks on the proof. A stronger version of this theorem, where $\mathcal{B}$ may be any complete Jordan algebra of operators, not necessarily commutative, can be found in [25, Theorem 2]. That proof relies on James's characterisation of weakly compact sets and the Bessaga-Pełczyński result that $X$ contains no copy of $c_{0}$ if and only if all series $\sum_{n} x_{n}$ in $X$ with $\sum_{n}\left|\left\langle x_{n}, x^{\prime}\right\rangle\right|$ convergent for all $x^{\prime} \in X^{\prime}$ are unconditionally norm convergent.

Corollary 6.2. Let $T$ be a normal operator on a Banach space $X$ that does not contain a subspace isomorphic to $c_{0}$. Then $T$ is a scalar-type spectral operator.

Proof. $T$ has a functional calculus (see $\S 2$ ) which, by the theorem, is weakly compact. Apply Theorem 6.1.

We can now present a theorem which is stronger than any other known to us in this area.

Theorem 6.3. Let $\mathcal{E}$ be a bounded Boolean algebra of hermitian projections on a Banach space $X$ and suppose that $X$ does not contain a subspace isomorphic to $c_{0}$. Then the weakly closed algebra $\overline{\mathcal{B}}^{w}$ generated by $\mathcal{E}$ is a $\mathrm{W}^{*}$-algebra and any faithful representation of $\overline{\mathcal{B}}^{w}$ as a concrete von Neumann algebra on a Hilbert space is $\mathrm{BWO}$ and $\mathrm{BSO}$ bicontinuous. Moreover, every operator in $\overline{\mathcal{B}}^{w}$ is a scalar-type spectral operator.

Proof. Theorem 6.1 shows that $\mathcal{E}$ is relatively weakly compact. The result follows from Theorem 3.6, Corollary 4.9, and Corollary 6.2. 
Corollary 6.4. Let $\mathcal{T}$ be a commuting family of scalar-type spectral operators on a Banach space $X$ that does not contain a subspace isomorphic to $c_{0}$. Suppose that the Boolean algebra generated by the resolutions of the identity of $T$ for each $T \in \mathcal{T}$ is uniformly bounded. Then every operator in the weakly closed ${ }^{*}$-algebra generated by $\mathcal{T}$ is a scalar-type spectral operator.

It has recently been shown [13, Theorem 2.5] that on a Banach lattice the Boolean algebra generated by two commuting bounded Boolean algebras of projections is itself bounded. Hence:

Corollary 6.5. Let $X$ be a complex Banach lattice not containing a copy of $c_{0}$, and let $\mathcal{T}$ be a finite commuting family of scalar-type spectral operators on $X$. Then every operator in the weakly closed ${ }^{*}$-algebra generated by $\mathcal{T}$ is a scalar-type spectral operator.

$c_{0}$ as the natural obstruction. If $X$ contains $c_{0}$ then there is a strongly closed bounded Boolean algebra $\mathcal{F}$ of projections on $X$ that is not complete $[\mathbf{1 2}$, Theorem 2]. Then the weakly closed algebra generated by $\mathcal{F}$ cannot have relatively weakly compact unit ball, and there can be no BWO bicontinuous faithful representation of this algebra on a Hilbert space.

\section{Boolean algebras with countable basis.}

As remarked above, $c_{0}$ seems to be the natural essential obstruction to extending the results of the previous section. It is of course conceivable that closer analysis will lead to a proof that the sum and product of a pair of commuting scalar-type spectral operators must be scalar-type spectral operators so long as the Boolean algebra generated by their resolutions of the identity is bounded.

We shall say that a Boolean algebra $\mathcal{E}$ has a countable basis if it contains a countable orthogonal subfamily $\mathcal{F}=\left(F_{m}\right)_{m \in \mathbb{N}}$ such that every $E \in \mathcal{E}$ can be written as the strong sum of a subset of this family. Note that then $I=\sum_{m=1}^{\infty} F_{m}$, the sum being strongly convergent.

Lemma 7.1. Let $\mathcal{C}$ be a commutative $\mathrm{C}^{*}$-algebra on $X$ and $\left(F_{m}\right)_{m \in \mathbb{N}}$ a countable family of positive elements of $\mathcal{C}$ such that $\sum_{m=1}^{\infty} F_{m}$ converges in the strong topology. Let $C_{m}$ be any sequence in $\mathcal{C}$ for which $0 \leq C_{m} \leq I(\forall m)$. Then

$$
\sum_{m=1}^{\infty} C_{m} F_{m}
$$

converges strongly. 
Proof. Note that $0 \leq C_{m} F_{m} \leq F_{m}(\forall m)$. Then, for $M<N$,

$$
0 \leq \sum_{m=M+1}^{N} C_{m} F_{m} \leq \sum_{m=M+1}^{N} F_{m} ;
$$

so, by Lemma 2.3 , the sequence $\left(C_{m} F_{m}\right)_{m=\in \mathbb{N}}$ is a strongly Cauchy sequence, and hence strongly convergent.

The following theorem generalises [13, Theorem 3.6]:

Theorem 7.2. Suppose that $\mathcal{E}^{(1)}$ and $\mathcal{E}^{(2)}$ are two commuting $\sigma$-complete Boolean algebras of projections on $X$ and that the Boolean algebra $\mathcal{E}$ generated by $\mathcal{E}^{(1)}$ and $\mathcal{E}^{(2)}$ is bounded. Assume, further, that $\mathcal{E}^{(2)}$ has a countable basis $\mathcal{F}=\left(F_{m}\right)_{m \in \mathbb{N}}$. Then $\mathcal{E}$ has a $\sigma$-complete extension, and hence a complete extension.

Proof. As remarked in $\S 3$ we may, and shall, assume that all the elements of $\mathcal{E}^{(1)}$ and $\mathcal{E}^{(2)}$ are hermitian. Let $\mathcal{C}$ be the weakly closed $\mathrm{C}^{*}$-algebra generated by $\mathcal{E}$.

For each sequence of projections $\left(E_{m}^{(1)}\right)_{m \in \mathbb{N}}$ taken from $\mathcal{E}^{(1)}$ we can, by Lemma 7.1, define $E=\sum_{m=1}^{\infty} E_{m}^{(1)} F_{m} \in \mathcal{C}$. Each such $E$ is a hermitian projection in $\mathcal{C}$ so has norm $\leq 1$.

Consider

$$
\mathcal{G} \triangleq\left\{\sum_{m=1}^{\infty} E_{m}^{(1)} F_{m}: E_{m}^{(1)} \in \mathcal{E}^{(1)}\right\} .
$$

It is clear that $F_{m} \in \mathcal{G}(\forall m)$, so $\mathcal{E}^{(2)} \subseteq \mathcal{G}$. Note also that for any $E^{(1)} \in \mathcal{E}^{(1)}$ we have $E^{(1)}=\sum_{m} E^{(1)} F_{m}$, so $E^{(1)} \in \mathcal{G}$. Thus $\mathcal{E}^{(1)} \vee \mathcal{E}^{(2)} \subseteq \mathcal{G}$.

It is clear that $\mathcal{G}$ is closed under products. Further, for any

$$
E=\sum_{m=1}^{\infty} E_{m}^{(1)} F_{m} \in \mathcal{G}
$$

we have

$$
I-E=\sum_{m=1}^{\infty}\left[I-E_{m}^{(1)}\right] F_{m} \in \mathcal{G},
$$

and so $\mathcal{G}$ is a Boolean algebra of hermitian projections on $X$.

Note that for any such $E \in \mathcal{G}$ we have $E F_{m}=E_{m}^{(1)} F_{m}(\forall m)$ : thus any element of $\mathcal{G}$, which can be written, though not in a unique manner, as an (orthogonal) sum

$$
E=\sum_{m=1}^{\infty} E_{m}^{(1)} F_{m}
$$

satisfies 


$$
E=\sum_{m=1}^{\infty} E_{m}^{(1)} F_{m}=\sum_{m=1}^{\infty} E F_{m} .
$$

Now consider a sequence $\left(E_{h}\right)_{h \in \mathbb{N}}$ of pairwise orthogonal projections in $\mathcal{G}$ :

$$
E_{h}=\sum_{m=1}^{\infty} E_{h, m}^{(1)} F_{m}=\sum_{m=1}^{\infty} E_{h} F_{m}
$$

For each $k$ and $m$ define

$$
G_{k, m} \triangleq \bigvee_{h=1}^{k} E_{h, m}^{(1)} \in \mathcal{E}^{(1)}
$$

and then define

$$
G_{m} \triangleq \bigvee_{k=1}^{\infty} G_{k, m}=\bigvee_{h=1}^{\infty} E_{h, m}^{(1)} \in \mathcal{E}^{(1)}
$$

Note that for each $k$ and $m$

$$
G_{k, m} F_{m}=\bigvee_{h=1}^{k} E_{h, m}^{(1)} F_{m}=\sum_{h=1}^{k} E_{h, m}^{(1)} F_{m}=\left(\sum_{h=1}^{k} E_{h}\right) F_{m}
$$

Suppose that $x \in X$ and $\varepsilon>0$. Then there exists an $M$ such that

$$
\left\|x-\sum_{m=1}^{M} F_{m} x\right\|<\varepsilon
$$

and so we can find $N$ such that for $1 \leq m \leq M$ and $k \geq N$

$$
\left\|\left(G_{m}-G_{k, m}\right) x\right\|<\varepsilon / M \text {. }
$$

Suppose that $j<k$ : then $0 \leq \sum_{h=j+1}^{k} E_{h} \leq I$, and so, by Lemma 2.3,

$$
\begin{aligned}
\left\|\left(\sum_{h=j+1}^{k} E_{h}\right) x\right\| \leq & \left\|\left(\sum_{h=j+1}^{k} E_{h}\right)\left(x-\sum_{m=1}^{M} F_{m} x\right)\right\| \\
& +\sum_{m=1}^{M}\left\|\left(\sum_{h=j+1}^{k} E_{h}\right) F_{m} x\right\| \\
\leq & \left\|x-\sum_{m=1}^{M} F_{m} x\right\|+\sum_{m=1}^{M}\left\|\left(G_{k, m}-G_{j, m}\right) F_{m} x\right\| \\
\leq & \left\|x-\sum_{m=1}^{M} F_{m} x\right\|+\sum_{m=1}^{M}\left\|\left(G_{k, m}-G_{j, m}\right) x\right\| \\
\leq & \varepsilon+\varepsilon=2 \varepsilon .
\end{aligned}
$$


This shows that $\mathcal{G}$ is $\sigma$-complete. Then $\overline{\mathcal{E}}^{s}$ is complete (Corollary 4.10).

From this we obtain the following results.

Theorem 7.3. Let $\mathcal{E}^{(1)}$ and $\mathcal{E}^{(2)}$ be two commuting $\sigma$-complete Boolean algebras of hermitian projections on $X$. Suppose that the Boolean algebra $\mathcal{E}$ generated by $\mathcal{E}^{(1)}$ and $\mathcal{E}^{(2)}$ is bounded, and that $\mathcal{E}^{(2)}$ has a countable basis. Then the weakly closed ${ }^{*}$-algebra $\mathcal{C}$ generated by $\mathcal{E}$ is a $\mathrm{W}^{*}$-algebra.

Corollary 7.4 (Extension of $[\mathbf{1 3}, 3.6]$ ). Let $X$ be a Banach space and $T_{1}$, $T_{2}$ be commuting scalar-type spectral operators on $X$ with resolutions of the identity $\mathcal{E}^{(1)}, \mathcal{E}^{(2)}$ such that $\mathcal{E}^{(1)} \vee \mathcal{E}^{(1)}$ is bounded. Suppose further that one of these operators has countable spectrum. Then all operators in the weakly closed ${ }^{*}$-algebra generated by $T_{1}$ and $T_{2}$ are scalar-type spectral operators.

\section{References}

[1] R.G. Bartle, N. Dunford and J.T. Schwartz, Weak compactness and vector measures, Canad. J. Math., 7 (1955), 289-305, MR 16,1123c, Zbl 0068.09301.

[2] C. Bessaga and A. Pełczyński, On bases and unconditional convergence of series in Banach spaces, Studia Math., 17 (1958), 151-164, MR 22 \#5872, Zbl 0084.09805.

[3] F.F. Bonsall and J. Duncan, Complete Normed Algebras, Springer-Verlag, 1973, MR 54 \#11013, Zbl 0271.46039.

[4] M.J. Crabb and P.G. Spain, Commutators and normal operators, Glasgow Math. J., 18 (1977), 197-198, MR 56 \#1115, Zbl 0351.47025.

[5] J. Diestel and J.J. Uhl Jr., Vector Measures, Amer. Math. Surveys, 15, Amer. Math. Soc., 1977, MR 56 \#12216, Zbl 0369.46039.

[6] H.R. Dowson, Spectral Theory of Linear Operators, Academic Press, 1978, MR 80c:47022, Zbl 0384.47001.

[7] H.R. Dowson and T.A. Gillespie, A representation theorem for a complete Boolean algebra of projections, Proc. Roy Soc. Edin., 83A (1979), 225-237, MR 80m:47039, Zbl 0417.47014.

[8] N. Dunford, Spectral operators, Pacific J. Math., 4 (1954), 321-354, MR 16,142d, Zbl 0056.34601.

[9] N. Dunford and J.T. Schwartz, Linear Operators. I. General Theory, Interscience, New York, 1958, MR 22 \#8302, Zbl 0084.10402.

[10] N. Dunford and J.T. Schwartz, Linear Operators, Part III: Spectral Operators, Interscience (Wiley), New York, 1971, MR 54 \#1009.

[11] S.R. Foguel, Sums and products of commuting spectral operators, Ark. Mat., 3 (1957), 449-461, MR 21 \#2914, Zbl 0081.12301.

[12] T.A. Gillespie, Strongly closed bounded Boolean algebras of projections, Glasgow Math. J., 22 (1981), 73-75, MR 82a:46018, Zbl 0455.47024.

[13] _ Boundedness criteria for Boolean algebras of projections, J. Functional Anal., 148 (1997), 70-85, MR 98h:47048, Zbl 0909.46017. 
[14] A. Grothendieck, Sur les applications linéaires faiblement compactes d'espaces du type $C(K)$, Canad. J. Math., 5 (1953), 129-173, MR 15,438b, Zbl 0050.10902.

[15] S. Kakutani, An example concerning uniform boundedness of spectral measures, Pacific J. Math., 4 (1954), 363-372, MR 16,143b, Zbl 0056.34702.

[16] S. Kantorovitz, On the characterization of spectral operators, Trans. Amer. Math. Soc., 110 (1964), 519-537, MR 28 \#3329, Zbl 0139.08702.

[17] I. Kluvanek, Characterization of scalar-type spectral operators, Arch. Math. (Brno), 2 (1966), 153-156, MR 35 \#2163, Zbl 0206.13704.

[18] T.W. Palmer, Unbounded normal operators on Banach spaces, Trans. Amer. Math. Soc., 133 (1968), 385-414, MR 37 \#6768, Zbl 0169.16901.

[19] G.K. Pedersen, $C^{*}$-Algebras and their Automorphism Groups, Academic Press, 1979, MR 81e:46037, Zbl 0416.46043.

[20] N.W. Pedersen, The resolutions of the identity for sums and products of commuting spectral operators, Math. Scand., 11 (1962), 123-130, MR 28 \#479.

[21] W. Ricker, Operator Algebras generated by Commuting Projections: A Vector Measure Approach, Lecture Notes in Mathematics, 1711, 1999, MR 2001b:47055, Zbl 0936.47020.

[22] P.G. Spain, On scalar-type spectral operators, Proc. Camb. Phil. Soc., 69 (1971), 409-410, MR 44 \#7338, Zbl 0211.44702.

[23] On commutative $\mathrm{V}^{*}$-algebras II, Glasgow Math. J., 13 (1972), 129-134, MR 47 \#7461, Zbl 0245.46099.

[24] - The $\mathrm{W}^{*}$-closure of a $\mathrm{V}^{*}$-algebra, J. London Math. Soc. (2), 7 (1973), 385-386, MR 49 \#9649, Zbl 0272.46047.

[25] _ A generalisation of a theorem of Grothendieck, Quarterly J. Math., 27 (1976), 475-479, MR 56 \#1007, Zbl 0341.46007.

Received October 1, 2001.

\author{
Mathematics Department \\ UNIVERSITY OF GLASGOW \\ Glasgow G12 8QW \\ SCOTLAND \\ E-mail address: hrd@maths.gla.ac.uk \\ Mathematics Department \\ BIRJAND UNIVERSITY \\ BIRJAND \\ IRAN \\ E-mail address: mohammadbg@yahoo.com \\ Mathematics Department \\ UNIVERSITY OF GLASGOW \\ GLASGOW G12 8QW \\ SCOTLAND \\ E-mail address: pgs@maths.gla.ac.uk
}

